\title{
Bibliografia sulla storia italiana degli anni ottanta
}

\section{Leonardo Casalino}

\section{OpenEdition}

\section{Journals}

Edizione digitale

URL: http://journals.openedition.org/cei/592

DOI: $10.4000 /$ cei.592

ISSN: 2260-779X

Editore

UGA Éditions/Université Grenoble Alpes

Edizione cartacea

Data di pubblicazione: 15 mars 2012

Paginazione: 299-303

ISBN: 978-2-84310-222-6

ISSN: 1770-9571

Notizia bibliografica digitale

Leonardo Casalino, «Bibliografia sulla storia italiana degli anni ottanta», Cahiers d'études italiennes [Online], 14 | 2012, online dal 15 septembre 2013, consultato il 28 mars 2021. URL: http:// journals.openedition.org/cei/592 ; DOI: https://doi.org/10.4000/cei.592 


\title{
BIBLIOGRAFIA SULLA STORIA ITALIANA \\ DEGLI ANNI OTTANTA
}

\author{
Leonardo Casalino
}

Université Stendhal - Grenoble 3

\section{Storie generali dell'Italia contemporanea}

Attal Frédéric, Histoire de l'Italie de 1943 à nos jours, Paris, Armand Colin, 2004.

Barbagallo Francesco (a cura di), Storia dell'Italia repubblicana, 3 vol., Einaudi, Torino I994-1997.

Bellucci Paolo e Segatti Paolo (a cura di), Votare in Italia: 1968-2008. Dall'appartenenza alla scelta, Il Mulino, Bologna, 2010.

Brice Catherine, Histoire de l'Italie, Paris, Perrin, 2003.

Casalino Leonardo e Giacone Alessandro, Manuale di storia politica dell'Italia repubblicana (dal 1946 ad oggi), Paris, Chemin de tr@verse, $201 \mathrm{~s}$.

Ciocca Pierluigi, Ricchi per sempre? Una storia economica d'Italia (I7962005), Torino, Bollati Boringhieri, 2007.

Colarizi Simona, Biografia della prima Repubblica 1946-I996, Roma-Bari, Laterza, 1996.

Crainz Guido, Autobiografia della Repubblica. Le radici dell'Italia attuale, Roma, Donzelli, 2009.

Craveri Pietro, La Repubblica dal 1958 al 1992, Torino, Utet, 1995.

Di Michele Andrea, Storia dell'Italia repubblicana (1948-2008), Milano, Garzanti, 2008.

Di Nolfo Ennio, La Repubblica delle speranze e degli inganni. L'Italia dalla caduta del fascismo al crollo della Democrazia cristiana, Firenze, Ponte delle Grazie, 1996.

Ginsborg Paul, Storia d'Italia (I943-1996), Famiglia, società, Stato, Torino, Einaudi, 1998. 
IsNenghi Mario (a cura di), I luoghi della memoria, 3 vol., Roma-Bari, Laterza, I997-I999.

Kogan Norman, Storia politica dell'Italia repubblicana, Roma-Bari, Laterza, I990 [1982].

LANaro Silvio, Storia dell'Italia repubblicana. L'economia, la politica, la cultura, la società dal dopoguerra agli anni 'oo, Venezia, Marsilio, 200I [1992].

Lazar Marc (a cura di), L'Italie contemporaine de 1945 à nos jours, Paris, Fayard, 2009.

Lepre Aurelio, Storia della prima Repubblica. L'Italia dal 1943 al 2003, Bologna, Il Mulino, 2004 [1993].

Lupo Salvatore, Partito e antipartito. Una storia politica della prima Repubblica (1946-78), Roma, Donzelli, 2004.

Mammarella Giuseppe, L'Italia contemporanea. I943-1998, Bologna, Il Mulino, 2000 [1985].

Milza Pierre, Histoire de l'Italie, Paris, Fayard, 2005.

Ridolf Maurizio, Storia politica dell'Italia repubblicana, Milano, Bruno Mondadori, 2010.

Sabbatucci Giovanni e Vidotto Vittorio (a cura di), Storia d'Italia,

- vol. 5, La Repubblica (1943-1963), Roma-Bari, Laterza, 1997.

- vol. 6, L'Italia contemporanea, Roma-Bari, Laterza, 1999.

Salvadori Massimo L., Storia d'Italia e crisi di regime. Saggio sulla politica italiana, I86I-2000, Bologna, Il Mulino, 200I.

Santarelli Enzo, Storia critica della Repubblica. L'Italia dal I945 al I994, Milano, Feltrinelli, 1996.

SCARPellini Emanuela, Dalla Bella Epoque al nuovo millenio, Roma-Bari, Laterza, 2008.

Scoppola Pietro, La repubblica dei partiti. Evoluzione e crisi di un sistema politico 1945-1996, Bologna, Il Mulino, I997 [I99I].

\section{Opere monografiche}

Aa. Vv, Il trionfo del privato, Roma-Bari, Laterza, I980.

Aa.Vv, Ripercorrere gli anni '8o, Censis (a cura di), Franco Angeli, Milano, I980.

Aa.Vv, Governare il cambiamento, atti della Conferenza programmatica di Rimini del PSI, 3I marzo-4 aprile I982, Roma, Il Compagno, I982. Amato Giuliano, Una Repubblica da riformare, Bologna, Il Mulino, I980. 
Ascoli Ugo e Catanzaro Raimondo (a cura di), La società italiana degli anni Ottanta, Roma-Bari, Laterza, 1988.

Ariemma Iginio, La casa brucia. I Democratici di sinistra dal PCI ai giorni nostri, Venezia, Marsilio, 2000.

Asor Rosa Alberto, La sinistra alla prova. Considerazioni sul ventennio 1976-1996, Torino, Einaudi, 1996.

Baldissera Alberto, La svolta dei quarantamila. Dai quadri Fiat ai Cobas, Milano, Edizioni di Comunità, 1988.

Barbagallo Francesco, Napoli fine Novecento. Politici, camorristi, imprenditori, Torino, Einaudi, 1997.

-, Enrico Berlinguer, Roma, Carocci, 2006.

Biasco Salvatore, «L'economia internazionale negli anni Ottanta. Rottura e continuità", in Storia dell'Italia repubblicana, vol. III, t. I, Torino, Einaudi, I996, pp. 25I-323.

Bidussa David (a cura di), Siamo Italiani, Milano, Chiarelettere, 2007. Bocca Giorgio, La disunità d'Italia, Milano, Garzanti, I990.

CAFAgna Luciano, La grande slavina. L'Italia verso la crisi della democrazia, Venezia, Marsilio, 1983.

Calabrese Omar, L'età neobarocca, Roma-Bari, Laterza, 1987.

Cazzola Franco, Della corruzione. Fisiologia e patologia di un sistema politico, Bologna, Il Mulino, 1988.

-, L'Italia del pizzo. Fenomenologia della tangente quotidiana, Torino, Einaudi, I992.

Colarizi Simona e al. (a cura di), Gli anni Ottanta come storia, Soveria Mannelli, Rubbettino, 2004.

Colarizi Simona e Gervasoni Marco, La cruna dell'ago. Craxi, il partito socialista e la crisi della Repubblica, Roma-Bari, Laterza, 2005.

Crainz Guido, Il paese mancato. Dal miracolo economico agli anni Ottanta, Roma, Donzelli, 2003.

Deaglio Enrico, Patria 1978-2008, Milano, Il Saggiatore, 2009.

-, Il raccolto rosso 1982-2010. Cronaca di una guerra di mafia e delle sue tristissime conseguenze, Milano, Il Saggiatore, 2010.

Debenedetti Franco e Pilati Antonio, La guerra dei trentanni. Politica e televisione in Italia 1975-2008, Torino, Einaudi, 2008.

De Lillo Antonio e Cavalli Alessandro (a cura di), Giovani anni '8o. Secondo rapporto Iard sulla condizione giovanile, Bologna, Il Mulino, 1988.

De Luna Giovanni, Figli di un benessere minore. La Lega 1979-1983, Scandicci (Firenze), La Nuova Italia, I994.

-, Le ragioni di un decennio 1969-1979. Militanza, violenza, sconfitta, memoria, Milano, Feltrinelli, 2009. 
De Felice Franco, «Nazione e crisi: le linee di frattura», in Storia dell'Italia repubblicana, vol. III, t. I, Torino, Einaudi, 1996, pp. 5-I27.

De Michele Stefano, I magnifici anni del riflusso. Come eravamo negli anni Ottanta, Venezia, Marsilio, 2003.

De Mita Ciriaco, Ragionando di politica. Le prospettive della democrazia italiana negli anni Ottanta, Milano, Rusconi, 1984.

Diamanti Ilvo, La Lega. Geografia, storia e sociologia di un nuovo soggetto politico, Roma, Donzelli, I995.

FoA Vittorio, Il cavallo e la torre, Torino, Einaudi, I99I.

Garzia Aldo, Da Natta a Natta. Storia del Manifesto e del PdUP, Bari, Edizioni Dedalo, 1985 .

Gervasoni Marco, Storia d'Italia degli anni Ottanta. Quando eravamo moderni, Venezia, Marsilio, $20 \mathrm{o}$.

Ginsborg Paul, Italy and Its discontents. Family, Civil Society, State: 1980200I, London, Penguin Books, 2001.

Ignazi Piero, Dal Pci al Pds, Bologna, Il Mulino, I992.

JaCobelli Jader (a cura di), Un'altra repubblica? Perché, come, quando, Roma, Bari-Laterza, 1988.

Jori Francesco, Dalla Liga alla Lega. Storia, movimenti, protagonisti, Venezia, Marsilio, 2009.

Lazar Marc, Maisons rouges. Les Partis communistes français et italien de la Libération à nos jours, Patis, Aubier, 1992.

Lerner Gad, Operai. La vita, la casa, le fabbriche di una classe che non c’è più, Milano, Feltrinelli, I988.

Lupo Salvatore, Storia della mafia, dall'origine ai giorni nostri, Roma, Donzelli, 2004.

Macaluso Emanuele, Leonardo Sciascia e i comunisti, Milano, Feltrinelli, 2010.

Mastropaolo Alfio, La Repubblica dei destini incrociati, Firenze, La Nuova Italia, 1996.

MAGri Lucio, Il sarto di Ulm. Una storia possibile del PCI, Milano, Il Saggiatore, 2009.

Menduni Enrico, Televisione e società italiana 1975-2000, Milano, Bompiani, 2002.

Millefiorini Andrea, La partecipazione politica in Italia. Impegno politico e azione collettiva negli anni Ottanta e Novanta, Roma, Carocci, 2002.

Moioli Vincenzo, Il tarlo delle leghe, Associazione culturale Antonio Gramsci (a cura di), Trezzo sull'Adda (MI), Comedit 2000, I99I.

Ortoleva Peppino, Un ventennio a colori. Televisione privata e società italiana 1975-2000, Firenze, Giunti, I995. 
PACI Massimo, Il mutamento della struttura sociale in Italia, Bologna, Il Mulino, 1992.

Pasquino Gianfranco (a cura di), Mass media e sistema politico, Milano, Franco Angeli, 1986.

Pintor Luigi, Parole al vento. Brevi cronache degli anni '8o, Milano, Kaos edizioni, I990.

Polo Gabriele e Sabbatini Claudio, Restaurazione italiana. I "35 giorni" alla Fiat del I980: passato e presente, Napoli-Roma, L'ancora, 2010.

Pons Silvio, Berlinguer e la fine del comunismo, Torino, Einaudi, 2006.

Rapini Andrea, Antifascismo e cittadinanza. Giovani, identità e memorie nell'Italia repubblicana, Bologna, Bonomia University Press, 2005.

Revelli Marco, Lavorare in Fiat, Milano, Garzanti, I989.

Ricolfi Luca e Sciolla Loredana, Senza padri né maestri. Inchiesta sugli orientamenti politici e culturali degli studenti, Bari, De Donato, I980.

Sabbatucci Giovanni, Il riformismo impossibile. Storie del socialismo italiano, Roma-Bari, Laterza, I99I.

Sangiovanni Andrea, Tute blu. La parabola operaia nell'Italia repubblicana, Roma, Donzelli, 2006.

Santomassimo Gianpasquale, "Origini e cultura del berlusconismo. L'eredità degli anni ottanta», Italia contemporanea, settembre 20IO, n. 260, pp. 383-391.

SARIo Beppe (a cura di), "Ritorno al futuro. Movimenti, culture e attivismo negli anni Ottanta», Zapruder, Gennaio-Aprile 2010.

SARTOR Nicola (a cura di), Il risanamento mancato. La politica di bilancio italiana, 1986-90, Roma, Carocci, I988.

Sylos Labini Paolo, Le classi sociali negli anni '8o, Roma-Bari, Laterza, I986.

Telese Luca, Qualcuno era comunista, Milano, Sperling \& Kupfer, 2009. Turani Giuseppe, 1985-1995: il secondo miracolo economico italiano, Milano, Sperling \& Kupfer, I986.

VACCA Giuseppe, Gorbačëv e la sinistra europea, Roma, Editori Riuniti, I989.

-, Ventanni dopo. La sinistra fra mutamenti e revisioni, Torino, Einaudi, I997. 
